\title{
Sustentabilidad del riego complementario en suelos Udipsament Típico y Hapludol Entico de Argentina
}

\author{
Mabel Vázquez, Pablo Gelati \& Guillermo Millán ${ }^{1}$
}

\begin{abstract}
RESUMEN
El riego complementario con aguas sódicas conduce a procesos dispersivos que deterioran el espacio poroso y con ello otras propiedades relacionadas. Esto podría producirse con bajos a medios niveles de RAS del agua, en suelos gruesos, cuando la illita domina la fracción arcilla. Se plantea la hipótesis de que el riego es capaz de provocar estados dispersivos en suelos de texturas medias a gruesas bajo estas condiciones, aun con aguas de baja a mediana sodicidad y períodos breves de la práctica, comprometiendo la sustentabilidad de sus sistemas productivos en el corto plazo. El objetivo de este trabajo es evaluar el grado de susceptibilidad a la dispersión y el efecto sobre la retención de agua y las propiedades hidráulicas de 2 suelos regados, Udipsament Típico y Hapludol Entico, en el Centro-E y NO, respectivamente, de la provincia de Buenos Aires, Argentina. La de agua en el suelo y los límites de Atterberg fueron superiores en las condiciones de riego en el suelo Udipsament Típico. La conductividad hidráulica disminuyó y el índice de dispersión fue superior para la situación de riego en ambos suelos. Estos resultados sugieren la no sustentabilidad del sistema de riego evaluado.
\end{abstract}

Palabras-clave: calidad de agua, salinización, índice de dispersión

\section{Complementary irrigation sustainability in Typic Udipsament and Entic Hapludol soils of Argentina}

\begin{abstract}
The complementary irrigation with high level of sodium in water leads to dispersive processes that deteriorate the porous space and therefore, other related properties. This could take place with low to medium levels of SAR of water, in coarse soil in which the illite is predominant in the clay fraction. It is hypothesized that irrigation may cause dispersive states in soils of medium to coarse texture under these circumstances, even with water containing low to medium levels of sodium and during short periods of use, compromising the sustainability of land production systems in the short term. The aim of this study was to evaluate the degree of susceptibility to dispersion and the effect on the soil water retention and its hydraulic properties of two irrigated soils, Typic Udipsament and Entic Hapludol, respectively, in the Center-East and North-West of Buenos Aires Province, Argentina. The soil water retention and the Atterberg's limits were higher under irrigated conditions in Typic Udipsament. The hydraulic conductivity decreased and the dispersion index was higher in the situation of irrigation in both soils. These results suggest the non sustainability of irrigation system evaluated.
\end{abstract}

Key words: quality water, salinization, dispersion index 


\section{INTRODUCCIÓN}

El riego complementario con aguas de naturaleza sódica y tecnologías que no contemplen medidas preventivas de control de los efectos adversos ocasionados, puede conducir a problemáticas de índole físico que disminuyen la capacidad productiva de los suelos.

La mayor parte de estas problemáticas tienen su origen en procesos dispersivos causados por altas concentraciones de $\mathrm{Na}^{+}$y una concentración electrolítica total por debajo de la "concentración de floculación crítica” definida por van Olphen (1977). En suelos esmectíticos, las arcillas saturadas por $\mathrm{Ca}^{2+}$ y $\mathrm{Mg}^{2+}$ forman tactoides o cuasi cristales en paquetes de 5 a 6 láminas con uniones de tipo cara-cara. En situaciones de enriquecimiento de $\mathrm{Na}^{+}$ a causa del agua de riego, el mismo se produce a expensas de la acidez de cambio, antes que por desplazamiento de $\mathrm{Ca}^{2+}$ (Pilatti et al., 2002; Marano \& Pilatti, 2002; Marano, 2004). Bajo estas circunstancias y con salinidad baja, la arquitectura mencionada se deteriora comprometiendo el espacio poroso, especialmente en detrimento de los poros de mayor tamaño y por ende de conducción del agua. Como consecuencia de estas modificaciones cabe esperar un aumento de la retención de agua y de otras variables relacionadas, como los límites líquido y plástico de Atterberg, así como una disminución de la infiltración y la conductividad hidráulica. Baumhardt et al. (1992) demostraron el efecto de la reducción drástica de la infiltración. Irurtia \& Mon (1998) y Geeves et al. (1998), entre otros muchos autores, afirmaron que la disminución de la permeabilidad por esta causa es de reversibilidad limitada. Churchman et al. (1993) señalaron que estos procesos pueden producirse aun a relativamente bajos niveles de relación de adsorción de $\mathrm{Na}^{+}$(RAS) del agua de riego, si la fracción arcilla está dominada por illita. Lebron et al. (1994) propusieron un índice de dispersión incluyendo a las fracciones arcilla y limo, de manera de predecir la vulnerabilidad de los suelos a estos procesos, demostrando la inclusión de otras fracciones granulométricas en el proceso dispersivo.

En el contexto de una gran difusión del riego complementario (SAGPyA, 1997) en la provincia de Buenos Aires, Argentina, con la connotación negativa de que gran parte de dichos suelos están dominados en la fracción arcilla por illita y otras esmectitas, se plantea la hipótesis de que el riego es capaz de provocar estados dispersivos en suelos de texturas medias a gruesas, aun con aguas de baja a mediana sodicidad y períodos breves de la práctica, comprometiendo la sustentabilidad de sus sistemas productivos en el corto plazo.

El objetivo de este trabajo es evaluar el grado de susceptibilidad a la dispersión y la afectación de las propiedades hídricas e hidráulicas de 2 suelos regados de textura arenoso-franca (Udipsament Tipico) y franca (Hapludol Entico), respectivamente, en el Centro-E y NO de la provincia de Buenos Aires.

\section{MATERIAL Y MÉTODOS}

\section{Características del área en estudio}

Este trabajo fue realizado en 2 suelos de la provincia de Buenos Aires, Argentina. El primero de ellos, clasificado como Udipsament Típico (UT) (Soil Survey Staff, 1999) del partido de 25 de Mayo, en la región Centro-E de la provincia, perteneciente a la Serie 25 de Mayo, de textura arenoso-franca (Carta de Suelos de la R. Argentina, Hoja 356027, Pueblitos-INTA, 1993). El segundo suelo es un Hapludol Entico (HE) del partido de Lincoln, en la zona NO, y pertenece a la Serie Santa Ana (1993), siendo el mismo de textura franca. Los suelos utilizados en este estudio se desarrollaron sobre loess, dominando la illita y la montmorillonita en la fracción arcilla. (Imbellone \& Teruggi, 1993; Giménez et al., 1996). Se trata de un área templada subhúmeda a humeda de precipitación media anual de 900-1000 mm, con déficits estivales y temperatura media anual de $16{ }^{\circ} \mathrm{C}$.

La antigüedad del riego fue de 4 años en el Udipsament Típico y de 3 años en Hapludol Entico. Se realizó mediante equipos de pivote central, con láminas que no superaron los $150 \mathrm{~mm}$ anuales.

\section{Aguas: muestreo y análisis}

Se tomaron muestras de agua de las respectivas perforaciones, con el recaudo de hacer circular la misma 2 h, antes de la extracción. Los análisis químicos se realizaron inmediatamente de llegadas al laboratorio.

Se determinó: $\mathrm{pH}$, conductividad eléctrica (CE), cationes $\left(\mathrm{Ca}^{2+}, \mathrm{Mg}^{2+}, \mathrm{Na}^{+}, \mathrm{K}^{+}\right)$y aniones $\left(\mathrm{CO}_{3}{ }^{2}, \mathrm{CO}_{3} \mathrm{H}^{-}, \mathrm{Cl}^{-}\right.$y $\left.\mathrm{SO}_{4}{ }^{2-}\right)$ (SAGPyA, 2004).

\section{Suelos: muestreo, análisis físicos y químicos}

Se muestreó el suelo “Testigo” y "Riego", fuera y dentro del área de los pivotes respectivamente. En cada lugar se extrajeron 9 muestras compuestas de 10 submuestras, a cada una de las siguientes profundidades: 0 a 20, 20 a 40 y 40 a $60 \mathrm{~cm}$. Se realizaron las siguientes determinaciones físicas:

a) límite plástico y líquido de Atterberg, índice de plasticidad (Forsythe, 1975) (LP, LL, IP)

b) retención de agua a 0,03 MPa y 1,5 MPa (Topp \& Reynold., 1992)

c) índice de dispersión (relación de \% de partículas comprendidas entre 2 y $63 \mathrm{~mm}$, sin y con dispersante) (profundidad de la muestra: 0 a $20 \mathrm{~cm}$ ) (Lebron et al., 1994) (ID)

d) conductividad hidráulica a flujo saturado (Topp \& Reynolds., 1992) (K)

e) índice de actividad de arcilla (LP / \% arcilla) (Resendiz, 1977)

Se llevó a cabo una caracterización general de los suelos (textura, materia orgánica (MO), pH, CE, capacidad de intercambio catiónica (CIC) y cationes de cambio). Las determinaciones químicas realizadas sobre el extracto de saturación de los suelos fueron las siguientes: CE, RAS, cationes $\left(\mathrm{Ca}^{2+}, \mathrm{Mg}^{2+}, \mathrm{Na}^{+}, \mathrm{K}^{+}\right)$y aniones $\left(\mathrm{CO}_{3}={ }^{2}, \mathrm{CO}_{3} \mathrm{H}^{-}, \mathrm{Cl}^{-}\right.$ $\left.\mathrm{y} \mathrm{SO}_{4}{ }^{2-}\right)($ SAGPyA, 2004) 


\section{Análisis estadístico}

La comparación de los resultados entre situaciones testigo y riego fue realizada a través del estadístico " $T$ ” para muestras apareadas. La asociación entre variables fue probada mediante correlaciones simples de Pearson (Mendenhall et al., 1986).

\section{RESULTADOS Y DISCUSIÓN}

Los resultados analíticos de caracterización general de los suelos se ilustran en la Tabla 1.

Dichos suelos poseen contenidos de MO medios, CIC baja a media, con una saturación de bases mayor al $80 \%$ y el complejo de cambio dominado por $\mathrm{Ca}^{2+}$ en ambos casos.

Las características de las aguas de riego y su calificación según diferentes criterios bibliográficos se presentan en la Tabla 2. Estos resultados permiten verificar que el agua empleada en el UT es calificada desde no salina a altamente salina, según el criterio, mientras que la del HE lo es desde no salina hasta medianamente salina. Su composición permite establecer que en ambos casos dominan el $\mathrm{NaCO}_{3} \mathrm{H} / \mathrm{Na}_{2} \mathrm{CO}_{3}$. La sodicidad evaluada a través del $\mathrm{pH}$, RAS y carbonato de sodio residual (CSR), indica que el agua utilizada en el UT puede ser calificada desde no sódica hasta predisponente a problemas crecientes de infiltración, y la del HE como no causante de problemas de pérdida de estructura hasta sódica. La relación $\mathrm{Mg}^{2+} / \mathrm{Ca}^{2+}$ es considerada no peligrosa por su incidencia en el proceso de sodificación en ambos casos, según antecedentes de Curtin \& Smille (1983).

La aplicación de los criterios de clasificación utilizados, seleccionados por su amplia difusión en Argentina, demuestra la disparidad de umbrales de identificación de las problemáticas de salinidad y sodicidad. Esta disparidad es originada por la diversidad de condiciones edafo-climáticas para las cuales fueron desarrollados dichos criterios y pone en evidencia la invalidez de su extrapolación, sin ajustes locales.

Las Figuras 1 y 2 muestran las propiedades físicas de los suelos UT y HE, en situaciones testigo y riego, respectivamente. En la Figura 1 se aprecia con claridad que la retención de agua en el suelo a ambas succiones es considerablemente mayor en la situación de riego respecto de la situación testigo (diferencia estadísticamente significativa $\mathrm{p}<0,01)$.
En la Tabla 3 se evalúa estadísticamente la asociación entre las propiedades físicas y las determinaciones químicas del extracto de saturación de los suelos estudiados.

Se verifican asociaciones estadísticamente significativas ( $\mathrm{p}<0,01)$ y de carácter positivo con el $\mathrm{pH}$ y el contenido de $\mathrm{CO}_{3} \mathrm{H}^{-}$, mostrando las relaciones causales de estas propiedades químicas. Esto sugeriría que el efecto dispersivo causado por el agua de riego de naturaleza bicarbonatada, a tra-

Tabla 2. Características generales de las aguas de riego. Diferentes calificaciones de aptitud

\begin{tabular}{|c|c|c|c|}
\hline & & \multicolumn{2}{|c|}{ Suelo regado } \\
\hline & & $\begin{array}{l}\text { Udipsament } \\
\text { Típico }\end{array}$ & $\begin{array}{c}\text { Hapludol } \\
\text { Éntico }\end{array}$ \\
\hline $\mathrm{pH}$ & & 8,2 & 8,3 \\
\hline CE & $\left(d S m^{-1}\right)$ & 0,8 & 0,7 \\
\hline $\mathrm{Ca}^{+2}$ & $\left(\mathrm{mmol}_{\mathrm{c}} \mathrm{L}^{-1}\right)$ & 0,8 & 0,7 \\
\hline$M g+2$ & $\left(\mathrm{mmol}_{\mathrm{c}} \mathrm{L}^{-1}\right)$ & 0,5 & 0,2 \\
\hline $\mathrm{Na}^{+}$ & $\left(\mathrm{mmol}_{\mathrm{c}} \mathrm{L}^{-1}\right)$ & 5,4 & 6,4 \\
\hline $\mathrm{K}^{+}$ & $\left(\mathrm{mmol}_{\mathrm{C}} \mathrm{L}^{-1}\right)$ & 0,3 & 0,2 \\
\hline $\mathrm{CO}_{3}=$ & $\left(\mathrm{mmol}_{\mathrm{C}} \mathrm{L}^{-1}\right)$ & 2,9 & 0,4 \\
\hline $\mathrm{CO}_{3} \mathrm{H}^{-}$ & $\left(\mathrm{mmol}_{\mathrm{c}} \mathrm{L}^{-1}\right)$ & 2,8 & 5,5 \\
\hline $\mathrm{Cl}^{-}$ & $\left(\mathrm{mmol}_{\mathrm{C}} \mathrm{L}^{-1}\right)$ & 1,2 & 1,1 \\
\hline $\mathrm{SO}_{4}=$ & $\left(\mathrm{mmol}_{\mathrm{c}} \mathrm{L}^{-1}\right)$ & 0,2 & 0,3 \\
\hline RAS & & 6,6 & 9,5 \\
\hline CSR & $\left(\mathrm{mmol}_{\mathrm{c}} \mathrm{L}^{-1}\right)$ & 4,3 & 5,0 \\
\hline $\mathrm{Mg}^{2+} / \mathrm{Ca}^{2+}$ & & 0,68 & 0,29 \\
\hline \multicolumn{4}{|l|}{ Calificaciones } \\
\hline Richards (1954) & & C2/C3S1 & $\mathrm{C} 2 \mathrm{~S} 2$ \\
\hline Rhoades (1972) & & C1S1 & C1S2 \\
\hline Ayers \& Wescot (1976) & & $\mathrm{C} 1 / \mathrm{C} 2 \mathrm{~S} 1$ & C1S1 \\
\hline Ayers \& Wescot (1987) & & C1/C2 S2 & C1S2 \\
\hline \multirow[t]{2}{*}{ Sing et al. (1996) } & & No salino & No salino \\
\hline & & No sódico & Sódico (CSR) \\
\hline \multirow[t]{4}{*}{ Richards (1954) } & \multicolumn{3}{|c|}{ C2: mediana salinidad } \\
\hline & \multicolumn{3}{|c|}{ C3: altamente salin } \\
\hline & \multicolumn{3}{|c|}{ S1: baja sodicidad } \\
\hline & \multicolumn{3}{|c|}{ S2: mediana sodicidad } \\
\hline \multirow[t]{3}{*}{ Rhoades (1972) } & \multicolumn{3}{|c|}{ C2:mediana salinidad } \\
\hline & \multicolumn{3}{|c|}{ S1: baja sodicidad } \\
\hline & \multicolumn{3}{|c|}{ S2: mediana modicidad } \\
\hline \multirow[t]{4}{*}{ Ayers \& Wescot (1976) } & \multicolumn{3}{|l|}{ C1: no salina } \\
\hline & \multicolumn{3}{|c|}{ C2: problemas crecientes de salinidad } \\
\hline & \multicolumn{3}{|c|}{ S1: sin probl. pérdida de estructura } \\
\hline & \multicolumn{3}{|c|}{ C1: baja salinidad } \\
\hline \multirow[t]{3}{*}{ Ayers \& Wescot (1987) } & \multicolumn{3}{|c|}{ C1: no salina } \\
\hline & \multicolumn{3}{|c|}{ C2: problemas crecientes de salinidad } \\
\hline & \multicolumn{3}{|c|}{ S2: problemas crecientes de infiltración } \\
\hline
\end{tabular}

Tabla 1. Caracterización general de los suelos estudiados

\begin{tabular}{|c|c|c|c|c|c|c|c|c|c|c|c|c|c|}
\hline \multirow{2}{*}{ Establecimiento } & \multirow{2}{*}{$\begin{array}{l}\text { Prof.(2) } \\
\text { (cm) }\end{array}$} & \multirow{2}{*}{$\begin{array}{c}\text { M.O. } \\
\left(g^{2} \mathrm{~kg}^{-1}\right)\end{array}$} & \multirow[t]{2}{*}{$\mathrm{pH}$} & CIC & $\mathrm{Ca}^{+2}$ & $\mathrm{Mg}+2$ & $\mathrm{Na}^{+}$ & $\mathrm{K}^{+}$ & \multirow{2}{*}{$\begin{array}{l}S^{(1)} \\
(\%)\end{array}$} & Arena & Limo & Arcilla & \multirow{2}{*}{ Clase textural } \\
\hline & & & & \multicolumn{5}{|c|}{$\left(\mathrm{cmol}_{\mathrm{c}} \mathrm{kg}^{-1}\right)$} & & & $(\%)$ & & \\
\hline \multirow[t]{3}{*}{ Udipsament típico } & 20 & 16,38 & 6,00 & 9,5 & 5,0 & 1,5 & 0,8 & 1,2 & 89,5 & 81 & 11 & 8 & arenoso franco \\
\hline & 40 & & & 8,5 & 5,1 & 0,9 & 0,8 & 1,0 & 91,8 & 81 & 10 & 9 & arenoso franco \\
\hline & 60 & & & 8,2 & 4,5 & 1,0 & 0,7 & 0,8 & 97,6 & 83 & 8 & 9 & arenoso franco \\
\hline \multirow[t]{3}{*}{ Hapludol éntico } & 20 & 21,67 & 5,90 & 14,0 & 8,3 & 2,2 & 0,7 & 1,4 & 90,0 & 48 & 34 & 18 & franco \\
\hline & 40 & & & 15,7 & 10,0 & 2,0 & 0,7 & 1,3 & 89,2 & 48 & 30 & 22 & franco \\
\hline & 60 & & & 14,9 & 9,5 & 2,2 & 0,7 & 1,2 & 91,3 & 46 & 30 & 24 & franco \\
\hline
\end{tabular}

(1) S: porcentaje de saturación de bases; (2) 20: 0 - $20 \mathrm{~cm} ; 40: 20-40 \mathrm{~cm} ; 60: 40$ - $60 \mathrm{~cm}$ 

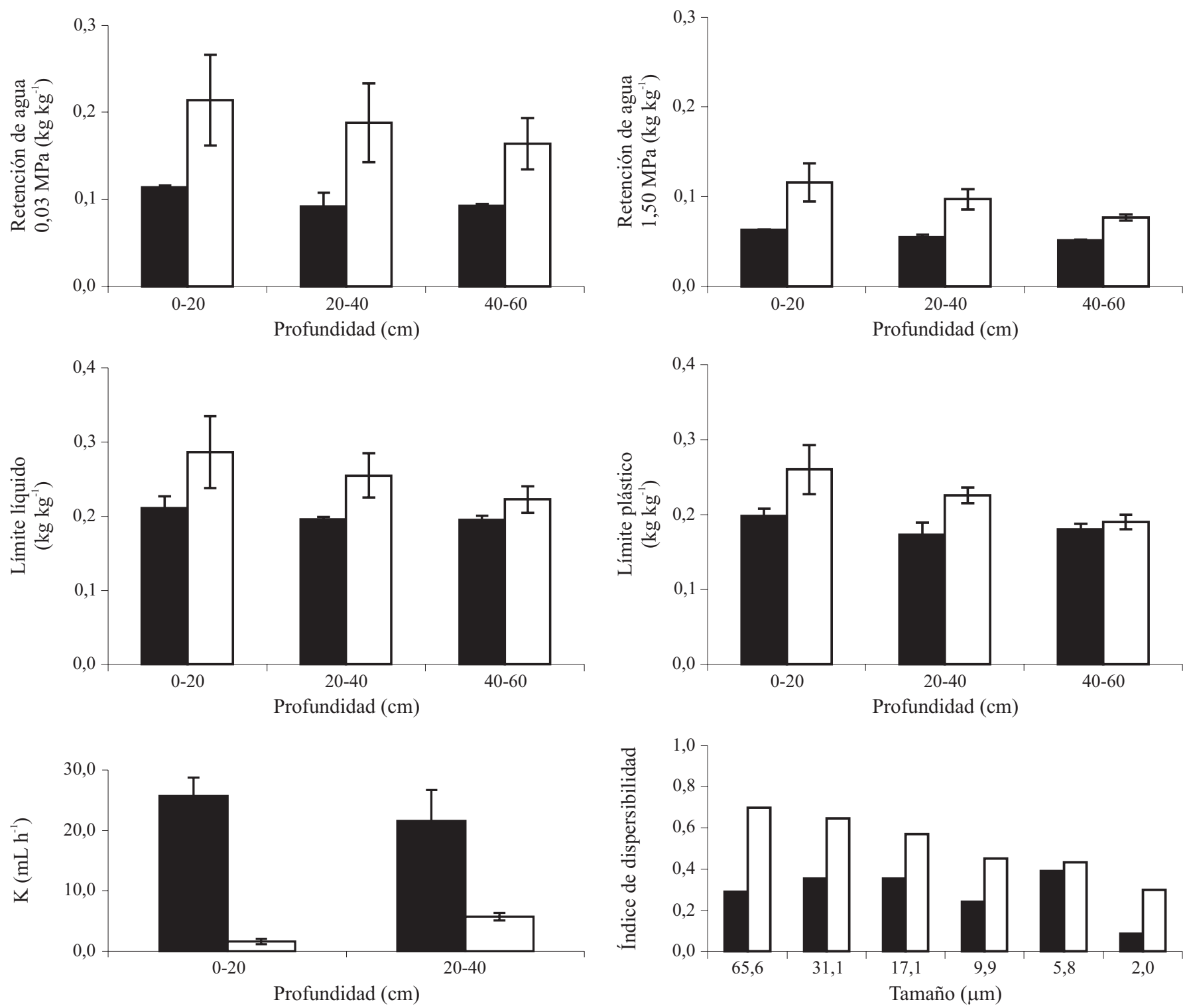

Testigo

$\square$ Riego

Figura 1. Retención de agua (0,03 MPa; $1,50 \mathrm{MPa})$, límites de Atterberg, conductividad hidráulica (K), e índice de dispersibilidad del suelo. Udipsament Típico

vés de la alteración del espacio poroso, podría ser responsable del aumento de la retención de agua. Resultados comparables pueden observarse en LP y LL. Como se sabe, estos límites ponen en evidencia fuerzas de cohesión y adhesión, resumidas en la propiedad denominada consistencia, afectada por la mineralogía de las partículas y su nivel de organización. Ambos límites tuvieron un comportamiento similar en UT, manifestando a las 3 profundidades evaluadas, mayores valores para las situaciones de riego, confirmando la alteración del espacio poroso (Figura 1). De la misma manera, en la Tabla 3 se verifican coeficientes de correlación estadísticamente significativos $(\mathrm{p}<0,01)$ y de carácter positivo con el $\mathrm{pH}$ y el contenido de $\mathrm{CO}_{3} \mathrm{H}^{-}$, para ambas variables. Consecuentemente $\mathrm{K}$ disminuye notablemente con el riego, pasando de valores superiores a $20 \mathrm{~mL} \mathrm{~h}^{-1}$ hasta cifras cercanas o inferiores a $5 \mathrm{~mL} \mathrm{~h}^{-1}$, de acuerdo a la profundidad (diferencia estadísticamente significativa $\mathrm{p}<0,01$ )
(Figura 1). Este comportamiento muestra una asociación estadísticamente significativa $(\mathrm{p}<0,05)$ y negativa con el contenido de $\mathrm{CO}_{3} \mathrm{H}^{-}$, confirmando la relación causal entre esta propiedad química y K, comentada precedentemente. Resultados similares obtuvieron Peinemann et al. (1998) estudiando suelos de diferentes áreas de la Pradera Pampeana, pertenecientes a los Grandes Grupos taxonómicos Hapludol y Argiudol, entre ellos del NO de la provincia de Buenos Aires, encontrando una disminución cercana al 50\% de K, tras la aplicación de 3 láminas de riego de 50 mm cada uno, a través de una simulación en permeámetros. En el pasado y aún recientemente, autores como Fitzpatrick et al. (1994), Jayawardene \& Chan (1994), Lebron et al. (1994), Ghiberto et al. (2002), demostraron ampliamente este fenómeno, causado por el hinchamiento y dispersión de las arcillas, cuando se producen enriquecimientos sódicos, aunque significativamente mayores a los demostrados en este trabajo. En el 

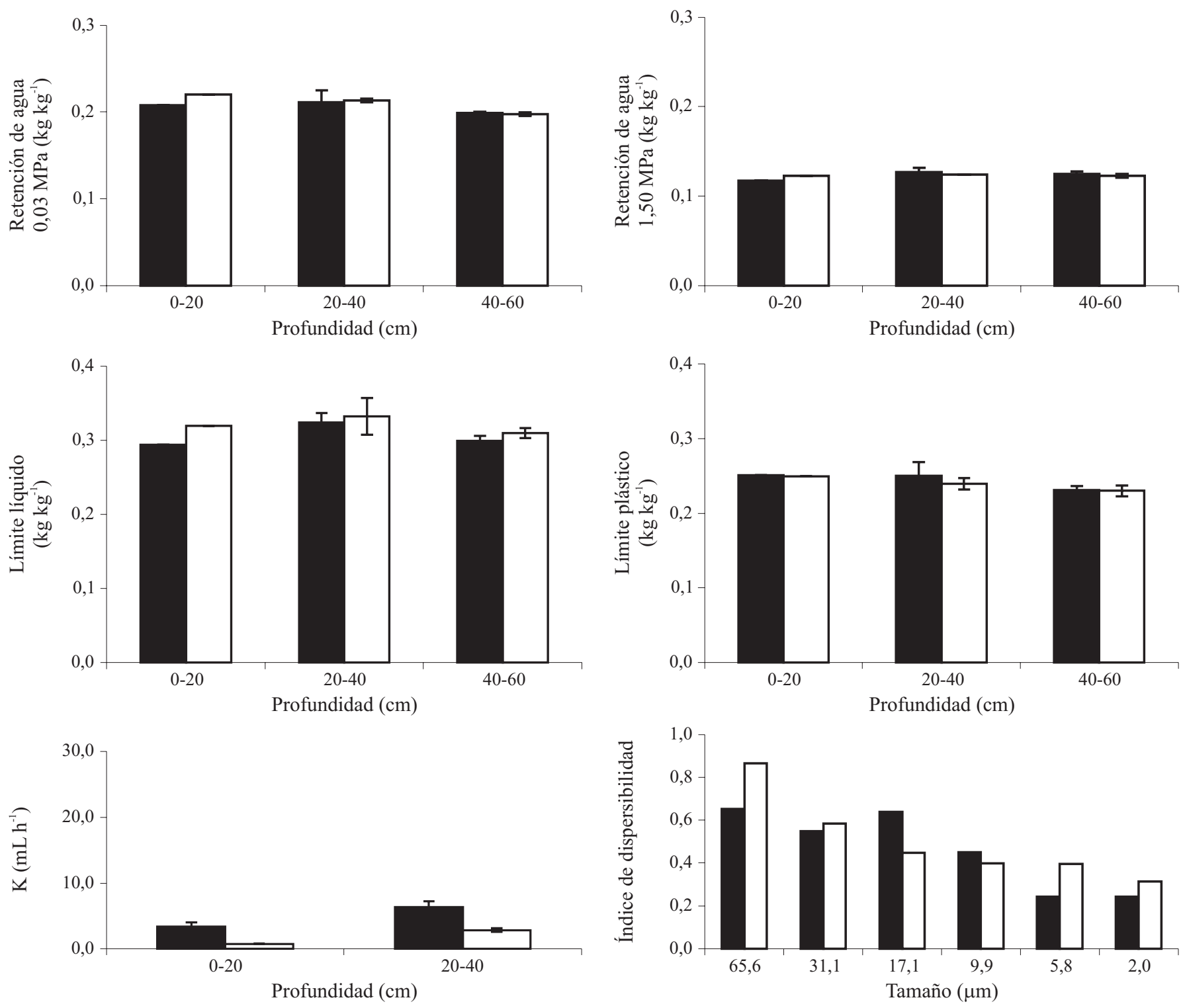

Testigo

$\square$ Riego

Figura 2. Retención hídrica (0,03 MPa; 1,50 MPa), límites de Atterberg, conductividad hidráulica (K), e índice de dispersibilidad del suelo. Hapludol Entico

HE (Figura 2), las diferencias fueron no significativas estadísticamente, tanto para las retenciones hídricas como LL y LP, mientras que se manifestaron diferencias en ID y K (diferencia estadísticamente significativa $\mathrm{p}<0,05$ ), aunque de menor magnitud que en el caso anterior.

A pesar que los índices de sodicidad son superiores en el agua usada en este suelo, la antigüedad de la práctica, las características granulométricas, una pluviometría superior para el período considerado y el contenido de materia orgánica, de acuerdo a antecedentes presentados por Andriulo (2002), podrían haber sido algunas de las causas de las diferencias entre los resultados de ambos suelos.

En la Figura 3 se presenta las asociaciones entre los límites de Atterberg y las retenciones hídricas a ambas succiones, en

Tabla 3. Coeficientes de correlación entre propiedades químicas (extracto de saturación) y físicas de los suelos

\begin{tabular}{|c|c|c|c|c|c|c|c|c|c|c|c|}
\hline & $\mathrm{pH}$ & CE & $\mathrm{Ca}^{2+}$ & $\mathrm{Mg}^{2+}$ & $\mathrm{Na}^{+}$ & $\mathrm{K}^{+}$ & $\mathrm{HCO}_{3}^{-}$ & $\mathrm{Cl}^{-}$ & $\mathrm{SO}_{4}=$ & RAS & K \\
\hline Límite líquido & 0,68 ** & 0,03 & 0,23 & 0,01 & 0,11 & 0,11 & $0,65^{\star *}$ & $-0,16$ & 0,1 & 0,11 & $-0,76^{* *}$ \\
\hline Límite plástico & 0,61 ** & 0,32 & $0,42^{*}$ & 0,24 & 0,30 & 0,39 & $0,85^{* *}$ & 0,06 & 0,35 & $-0,16$ & $-0,67^{* *}$ \\
\hline Ret. de agua. $0,03 \mathrm{MPa}$ & 0,66 ** & 0,13 & 0,02 & $-0,07$ & 0,31 & 0,13 & 0,64 ** & $-0,08$ & 0,23 & 0,33 & $-0,87^{\star *}$ \\
\hline Ret. de agua. $1,50 \mathrm{MPa}$ & 0,67 ** & 0,06 & 0,29 & 0,05 & 0,13 & 0,11 & $0,51 * *$ & $-0,08$ & 0,12 & 0,11 & $-0,82^{\star *}$ \\
\hline K & $-0,37$ & $-0,04$ & $-0,25$ & 0,26 & $-0,28$ & 0,08 & $0,35^{*}$ & $-0,09$ & $-0,21$ & $-0,30$ & - \\
\hline
\end{tabular}


el suelo UT. La relación entre estas propiedades hídricas indica que el suelo regado posee mayor retención de agua a ambas succiones y esto se asocia con mayores volúmenes de agua de los límites líquido y plástico del suelo, con la consecuente alteración de su dinámica. La significancia estadística de los coeficientes de correlación entre $\mathrm{K}$ y las variables retención de agua (0,03 y 1,5 Mpa) y límites de Atterberg, de carácter negativo para el conjunto de situaciones evaluadas en ambos suelos (testigo y riego), corrobora esta afirmación (Tabla 3).

La Figura 4 presenta la relación entre los límites de Atterberg y las retenciones hídricas a ambas succiones, en el suelo HE. En este caso y concordantemente con la ausencia de afectación de las variables de retención de agua y límites de Atterberg a causa del riego, no se evidencia asociación entre las mencionadas variables.
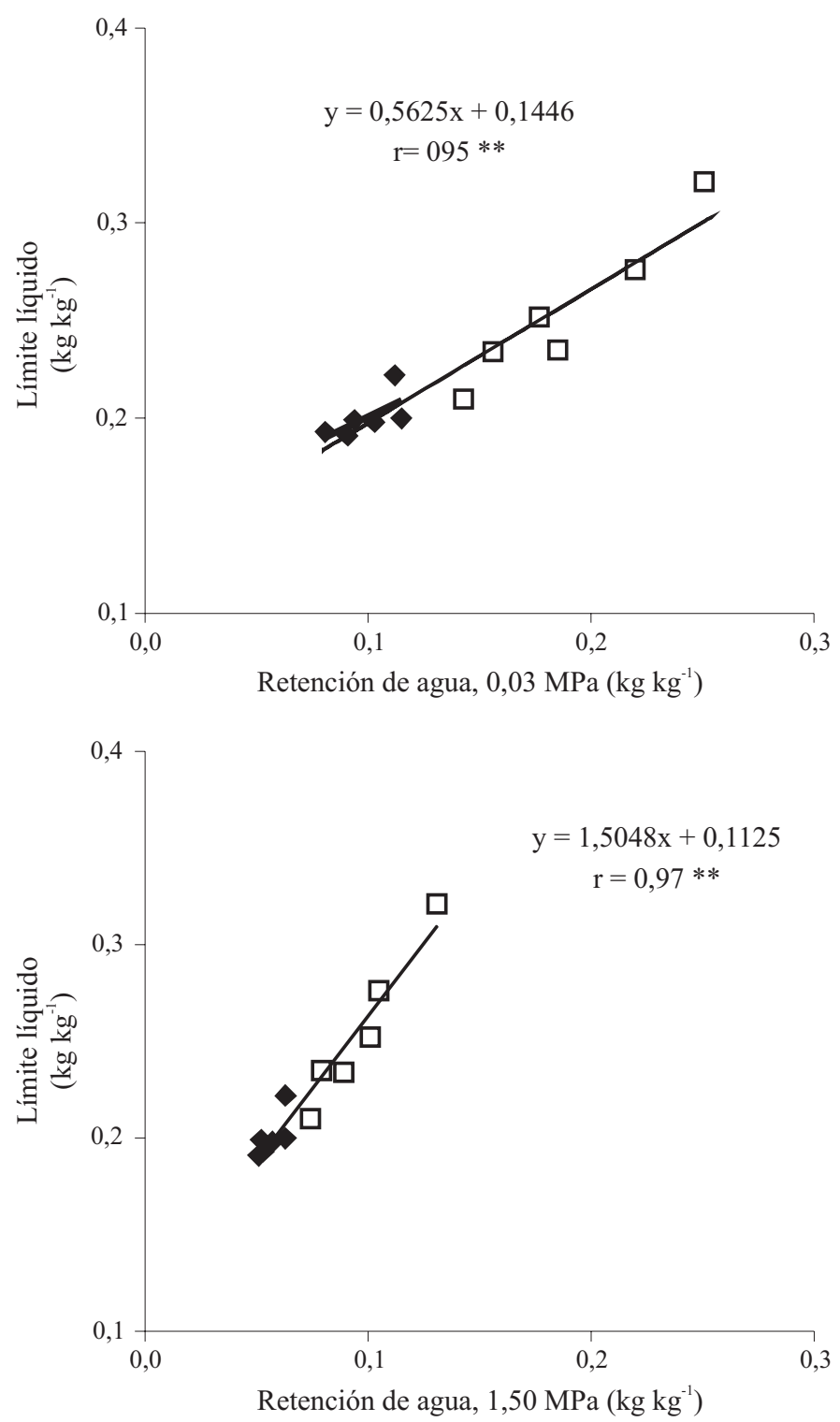

Cabe acotar que ya tempranamente en el desarrollo de esta temática, Gibbs (1945) señaló que suelos con índices de plasticidad (IP) $<1 \mathrm{~kg} \mathrm{~kg}^{-1}$ y límite líquido (LL) $<0,300 \mathrm{~kg} \mathrm{~kg}^{-1}$ son erodables.

Estos conceptos fueron confirmados con posterioridad (Cole \& Lewis, 1960; Gibbs, 1983). Los suelos UT (IP: 0,015 kg kg-1; LL: 0,200 $\mathrm{kg} \mathrm{kg}^{-1}$ ) y HE (IP: 0,074 $\mathrm{kg} \mathrm{kg}^{-1}$; LL: $0,300 \mathrm{~kg} \mathrm{~kg}^{-1}$ ) estudiados, alcanzan estos umbrales, de manera que su susceptibilidad a la erosión los condiciona a padecer la problemática con mayor facilidad ante sucesos de sodificación causados por el riego. Resendiz (1977) afirmó que suelos con índices de actividad de arcilla entre 0,003 y 0,01 son susceptibles a dispersión y erosión. Este índice alcanza valores de 0,003 en UT y 0,004 en HE, reafirmando el concepto de susceptibilidad de los suelos estudiados.
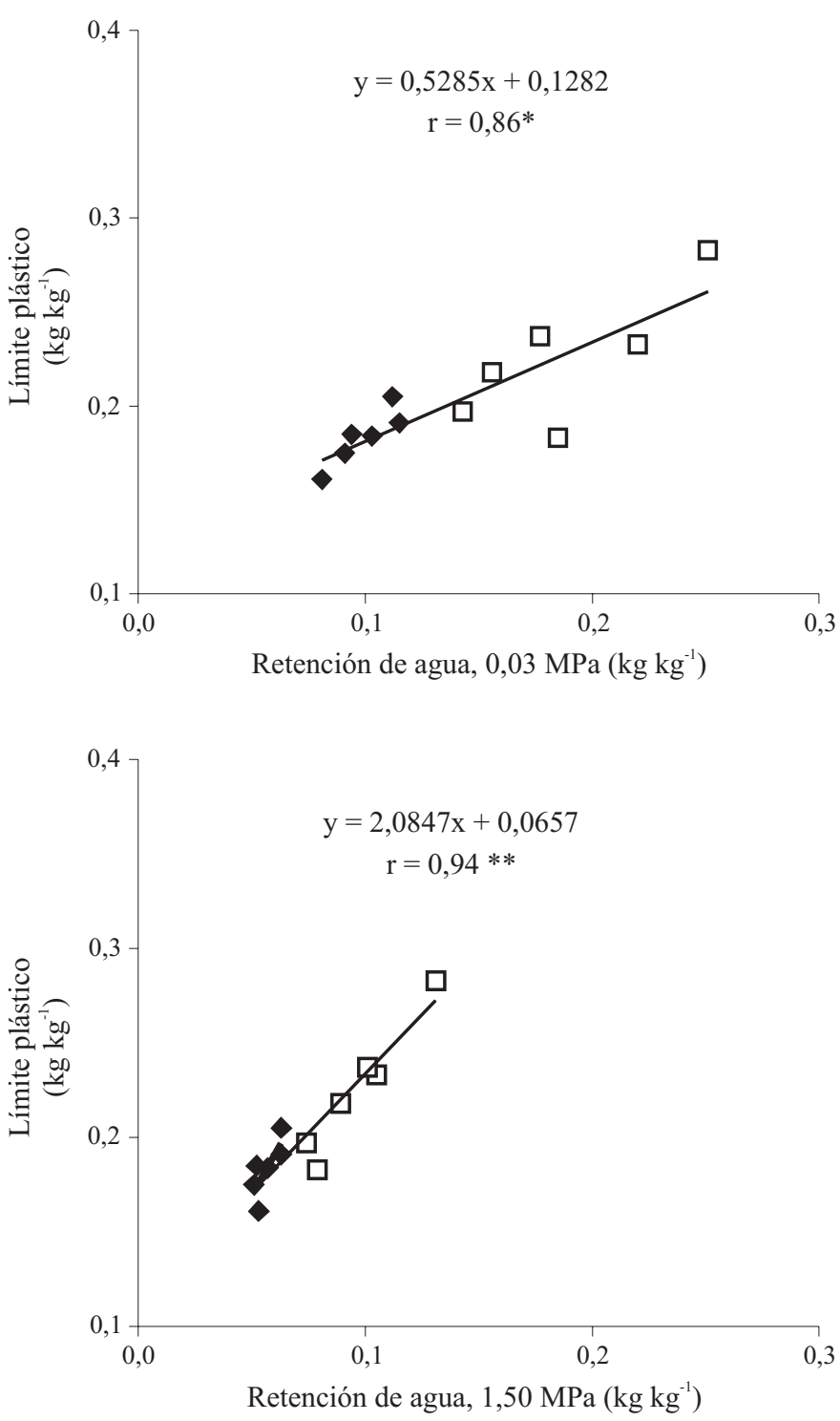

Riego

Figura 3. Asociación entre límites de Atterberg y retenciones hídricas a 2 succiones, en suelo Udipsament Típico, con y sin riego 

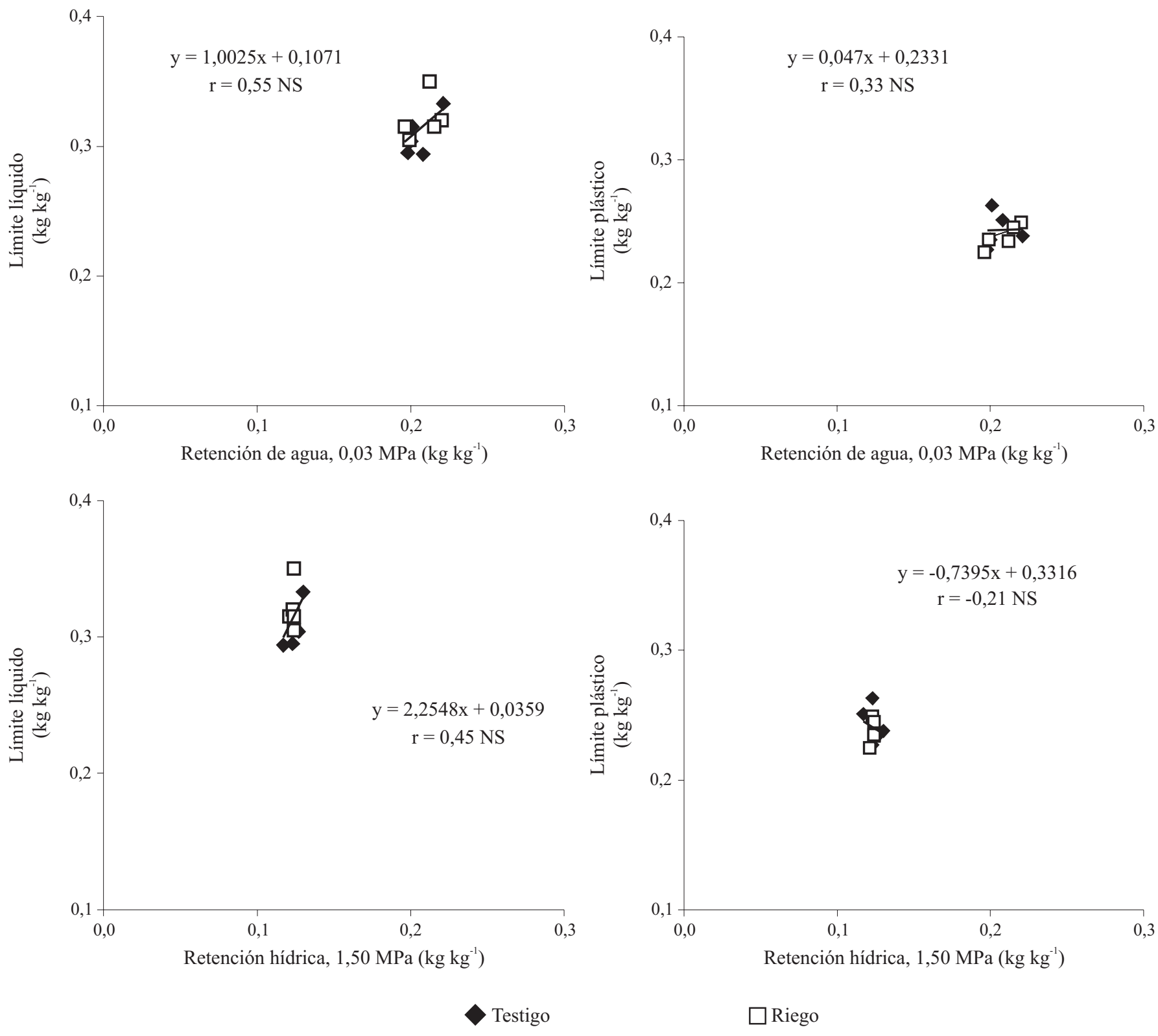

Figura 4. Asociación entre límites de Atterberg y retenciones hídricas a 2 succiones, en suelo Hapludol Éntico, con y sin riego

Lebron et al. (1994) propusieron el uso de un índice de dispersión de arcillas para clasificar la susceptibilidad a la erosión de los suelos. Según estos autores, el índice de dispersión estaría constituido por el cociente entre el porcentaje de arcilla evaluado por métodos de sedimentación, sin y con dispersante. Simultáneamente, los mismos autores, introdujeron el concepto de dispersión para tamaños de partícula no sólo de la categoría de arcilla $(<2 \mathrm{~mm})$ si no también de arcilla más limo fino $(<6,3 \mathrm{~mm})$, por considerar que este tamaño de partícula podría estar involucrado en procesos dispersivos. La Figura 5 ilustra los resultados obtenidos en UT, discriminadamente para 2 profundidades de muestreo, 0-20 (A, B) y 20-40 cm (C, D), hasta el tamaño de partícula de arena fina $(63 \mathrm{~mm})$.

El contenido de partículas fue superior en las situaciones de riego respecto de los testigos a ambas profundidades de muestreo, tanto en los procedimientos "sin dispersante" (Fi- gura 5 A, C) como “con dispersante” (Figura 5 B, D) (dif. est. sign. $\mathrm{p}<0,05)$.

Estos resultados demostrarían diferentes aspectos. En primer lugar que el proceso dispersivo ya ha ocurrido, en segundo lugar que tal fenómeno involucra a tamaños de partícula o pseudopartículas comprendidos entre arcilla y arena fina, no considerados por Lebron et al. (1994). Así mismo dicha dispersión ocurrió tanto en la capa superficial como subsuperficial.

Por otro lado el aumento del ID (diferencia estadísticamente significativa $\mathrm{p}<0,01$ ) (Figura 1) demuestra claramente que aun en suelos de textura relativamente gruesa como el presente, con dominio de illita en la fracción fina, los procesos dispersivos potenciales causados por el agua de riego, podrían ser aun mayores, como consecuencia de la modificación del tamaño de partículas medido cuando se agrega el dispersante en la determinación densimétrica. Este 

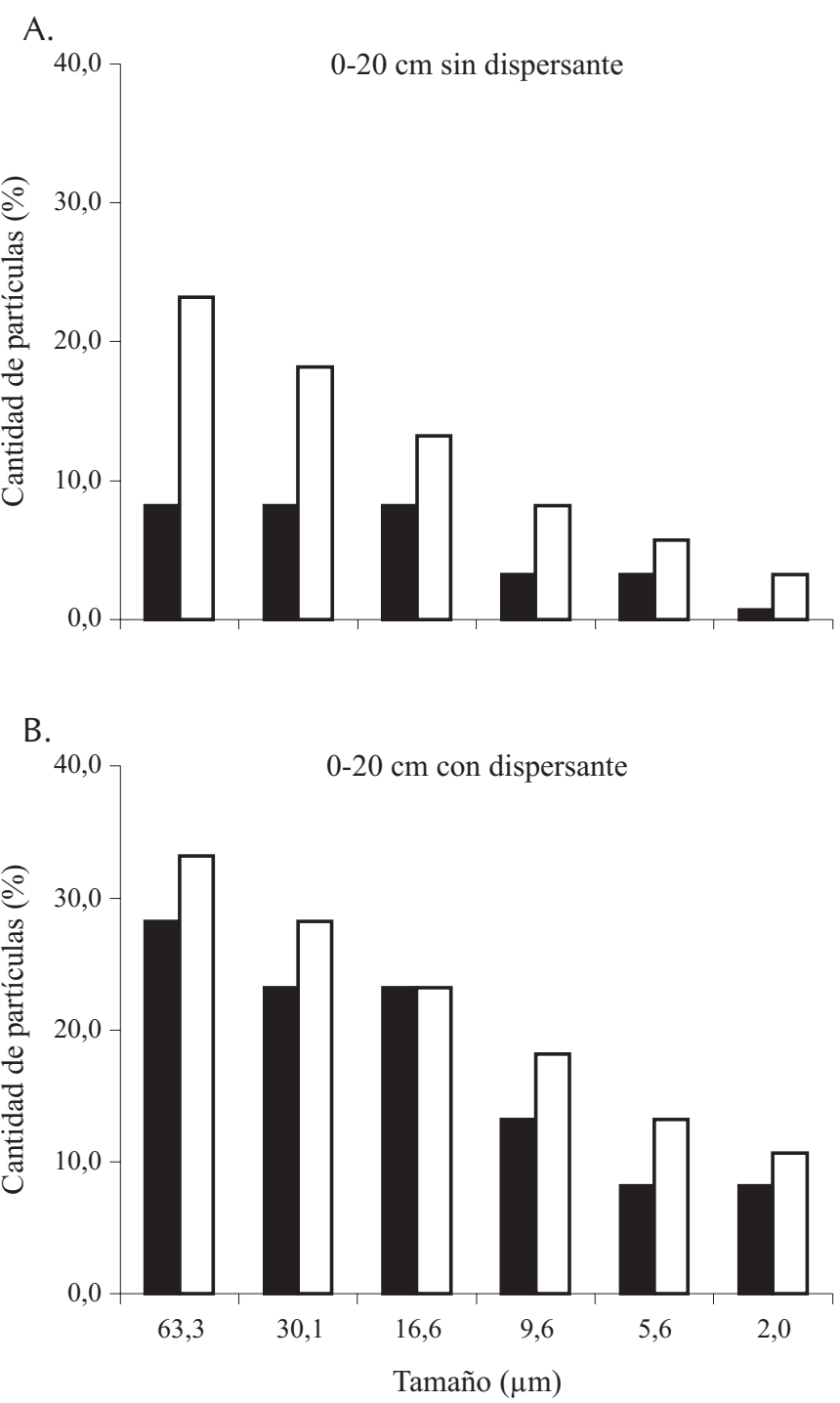

C.

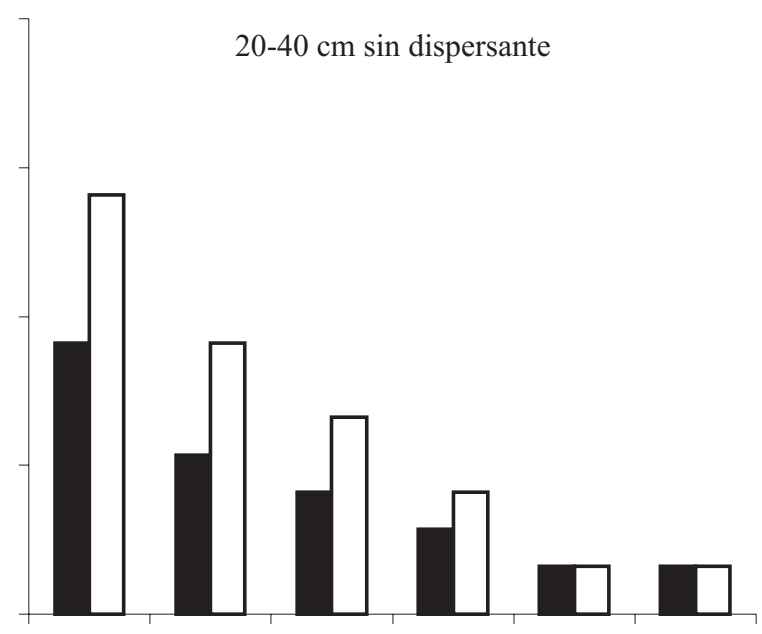

D.

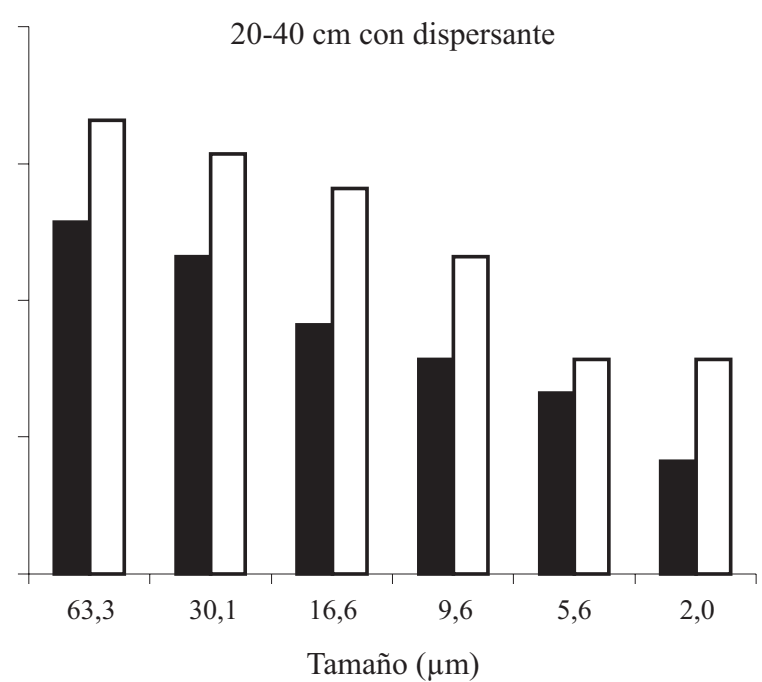

Testigo

Riego

Figura 5. Porcentaje de partículas de diferentes tamaños (con/sin dispersante) a 2 profundidades $(0-20 / 20-40 \mathrm{~cm})$ en situaciones testigo y riego del suelo. Udipsament Típico

fenómeno permite diagnosticar acontecimientos similares de continuar el riego con agua de la misma calidad que la usada hasta el presente. Esta última consideración sería la consecuencia de la mayor debilidad de unión entre las partículas en las situaciones de riego, demostrando una mayor potencialidad de la problemática en el futuro.

Abu-Sharar et al. (1987 a y b) explicaron la razón de la inclusión de la fracción limo en la determinación del índice de dispersión de arcilla. Estos autores afirmaron que la ruptura de los agregados del suelo en microagregados del tamaño limo, podría ser el paso previo a la dispersión de arcillas. Los resultados aquí encontrados confirmarían que este es un proceso jerárquico a partir de tamaños de agregados superiores a los citados por estos autores, frente al concepto de implosión a partículas individuales postulado por Rahman et al. (1996). Así mismo justificarían la afectación de la estabilidad estructural causada por el riego complementario en suelos de diferentes regiones (Baker et al., 2004; Raine, 2004).

En la Figura 6 se observa la relación entre la CE y el RAS entre las situaciones Testigo y Riego en ambos suelos (UT: A, B; HE: C, D). Cabe destacar que si bien el riego produjo un aumento estadísticamente significativo de la sodicidad ( $<<0,05)$, los fenómenos físicos descriptos se manifestaron claramente afectados a valores relativamente bajos de RAS, de acuerdo a criterios bibliográficos desarrollados en otros ambientes edafo-climáticos (Ayers \& Wescot, 1987). Esto relativiza la afirmación de Génova $(2002,2005)$ respecto de la resiliencia de suelos bonaerenses a los procesos de sodificación causados por el riego, exclusivamente a través de parámetros químicos.

Dados los resultados obtenidos, la ejecución continuada del riego complementario en las condiciones edafo-climáticas y tecnológicas empleadas en ambos suelos estudiados, 
A.

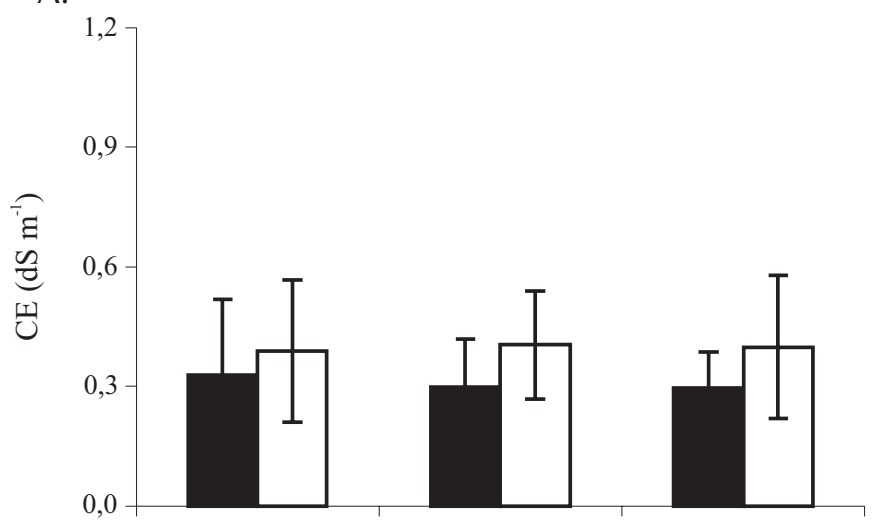

B.

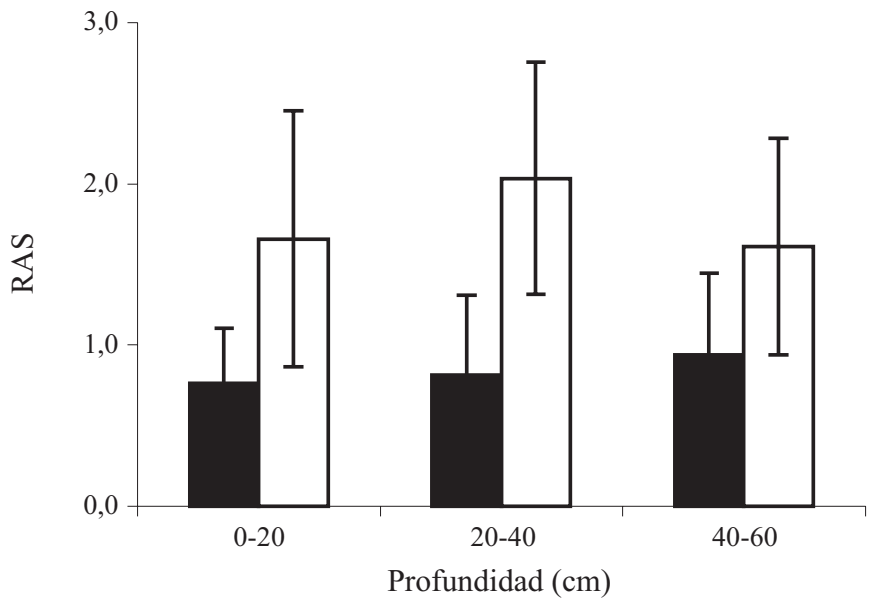

C.

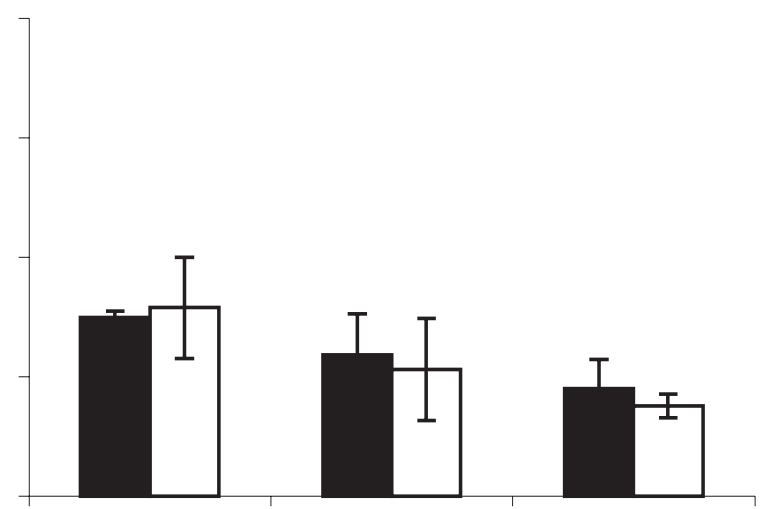

D.

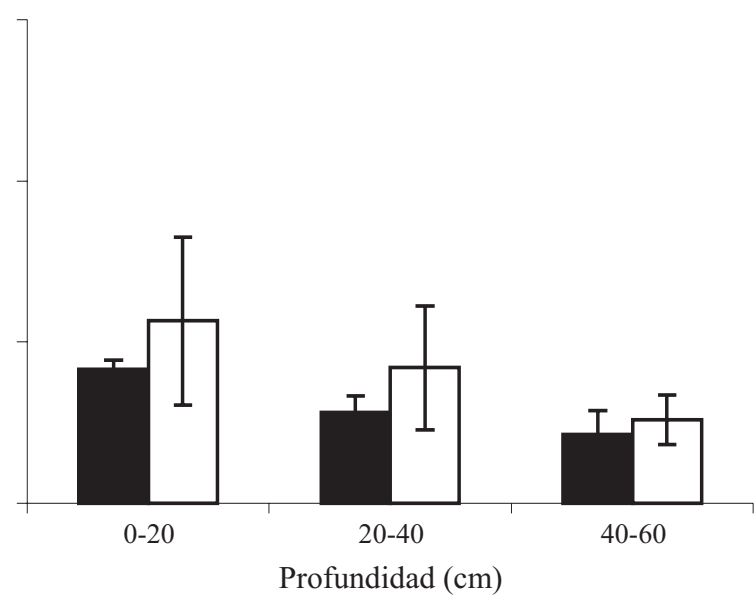

Testigo

Riego

coincidentes con la realidad regional de la práctica, puede definirse como un sistema no sustentable de producción, aunque de connotaciones particulares en cada caso.

\section{CONCLUSIONES}

1. En el Udipsament Típico aumentó la retención de agua a succiones de 0,03 y 1,50 MPa, los límites líquido y plástico de Atterberg y el índice de dispersión hasta tamaño de partículas y/o pseudopartículas de arena fina. Disminución de la conductividad hidráulica a flujo saturado. Las variaciones previamente descriptas se asociaron con el $\mathrm{pH}$ y el contenido de $\mathrm{CO}_{3} \mathrm{H}^{-}$del extracto de saturación del suelo, las que se producen a valores de la RAS menores a 2 .

2. En el Hapludol Entico disminuye la conductividad hidráulica a flujo saturado y el índice de dispersión hasta tamaño de arena fina, a pesar de no haberse registrado diferencias estadísticamente significativas en los parámetros hídricos (retención de agua, límites líquido y plástico de Atterberg). Estos cambios se produjeron a valores de la RAS menores a 1,2 .

3. Los resultados de los índices de actividad de arcilla y dispersión, permitieron comprobar la potencialidad de los suelos de padecer mayores procesos dispersivos que los evaluados hasta el presente, señalando en conjunto, la no sustentabilidad de la práctica de riego en los sistemas estudiados.

\section{LITERATURA CITADA}

Abu-Sharar, T. M.; Bingham, F. T.; Rhoades, J. D. Stability of soil aggregates as affected by electrolyte concentration and composition. Soil Science Society American Journal, Madison, v.51, n.2, p.309-314, 1987a.

Abu-Sharar, T. M.; Bingham, F. T.; Rhoades, J. D. Reduction in hydraulic conductivity in relation to clay dispersion and disaggregation. Soil Science Society American Journal, Madison, v.51, n.2, p.342-346, 1987b.

Andriulo, A.; Bueno, M.; de Orellana, J.; Sasal, C.; Rimatori, F. Efecto de la relación de adsorción de sodio del agua sobre la estabilidad de agregados. En: Congreso Argentino de la Ciencia del Suelo, 18, 2002, Puerto Madryn. Actas... Puerto Madryn: Asociación Argentina de la Ciencia del Suelo, 2002. CD Rom. 
Ayers, R. S.; Wescot, D. W. Calidad del agua para la agricultura. Roma: FAO, 1976. 85p. Riego y Drenaje. N 29

Ayers, R. S.; Wescot, D. W. Calidad del agua en la agricultura. Roma: FAO: Riego y Drenaje. No 29, Roma. 1987. 173p.

Baker, B. J.; Fausey, N. R.; Islam, K. R. Comparison of soil physical propierties under two different water table management regimes. Soil Science Society American Journal, Madison, v.68, n.6, p.1973-1981. 2004.

Baumhardt, R. L.; Wendt, C. W.; Moore, J. Infiltration in response to water quality, tillage, and gypsum. Soil Science Society American Journal, Madison, v.56, n.1, p.261-266, 1992.

Cole, D. C. H.; Lewis, J. G. Piping failure of earthen dams built of plastic materials in arid climates. En New Zealand Conference Soil Mechanics, 1960, Sydney. Proceeding... Sydney: Foundation Enginery/Institute Enginery. 1960, p.93-99.

Churchman, G. J.; Skjemstad, O.; Oades, M. J. Influence of clay minerals and organic matter on effects of sodicity on soils. Australian Journal Soil Research, Victoria, v.31, n.6, p.779800, 1993.

Curtin, D.; Smillie, G. W. Soil solution composition as affected by liming and incubation. Soil Science Society American Journal, Madison, v.47, p.701-707, 1983.

Eaton, F. M. Significance of carbonates in irrigation waters. Soil Science, Baltimor, v.69, n.1, p.123-133, 1950.

Fitzpatrick, R. W.; Boucher, S. C.; Naidu, R.; Fritsch E. Environmental consequences of soil sodicity. Australian Journal Soil Research, Victoria, v.32, n.5, p.1069-1093, 1994.

Forsythe, W. Física de suelos. Manual de lboratorio. San José, Costa Rica: IICA, 1975. cap. 5 e 6, p.439-68.

Geeves, G. W.; Cresswell, H. P.; Murphy, B. W. Two indices of soil structure based on prediction of soil water processes. Soil Science Society American Journal, Madison, v.62, n.1, p.223232, 1998.

Génova, L. Resistencia y resiliencia de 6 Argiudoles pampeanos a las degradaciones salina y sódicas, regados complementariamente con agua subterránea. En: Congreso Argentino de la Ciencia del Suelo, 18, 2002. Puerto Madryn. Actas... Puerto Madryn: Asociación Argentina de la Ciencia del Suelo, 2002. CD Rom.

Génova, L. Sostenibilidad de agroecosistemas pampeanos regados complementariamente. En: Congreso Argentino de Ingeniería Rural, 8, Merlo, San Luis, Argentina. 2005. Actas... Merlo: Asociación Argentina de Ingeniería Rural, 2005, CD Rom.

Ghiberto, P. J.; Marano, R. P.; Pilatti, M. A.; Felli, O. M. Riego suplementario con aguas bicarbonatadas sódicas en Molisoles de Santa Fe. Estudio de caso: Degradación física en un Argiudol en el Centro-E santafesino. En: Congreso Argentino de la Ciencia del Suelo, 18, 2002, Puerto Madryn. Actas... Puerto Madryn: Asociación Argentina de la Ciencia del Suelo, 2002. CD Rom.

Gibbs, H. S. Tunnel-gully erosion on the Wither Hills, Marlborough, New Zealand. New Zealand Journal Science Technology, Victoria, v.27, p.133-146, 1945.

Gibbs, H. S. Effect of natural organic coatings on the coagulation of particles. Environmental Science Technology, Columbus, v.17, p.237-240, 1983.
Giménez, J. E.; Imbellone, P. A.; Iasi, R. R.. Suelos con fragipan del noroeste de la Provincia de Buenos Aires. Revista del Museo de La Plata (NS) Geología XI, La Plata, v.109, n.11, p.119-134. 1996.

Imbellone, P. A.; Teruggi, M. E. Paleosols in loess deposits of the Argentine Pampas. Quaternary International, Pergamon, v.17, p.49-55, 1993.

INTA - Instituto Nacional de Tecnología Agropecuária. Carta de Suelos de la Republica Argentina, Diego de Alvear: INTA,1993. Hoja 3563-11.

Irurtia, C. B.; Mon, R. Cambios en las propiedades físicas y químicas de los suelos de la región pampeana después de cinco años de riego suplementario. En: Congreso Argentino de la Ciencia del Suelo, 16, 1998, Carlos Paz. Actas... Carlos Paz: Asociación Argentina de la Ciencia del Suelo, 1998, p.241-242.

Jayawardene, N. S.; Chan, K. Y. The management of soil physical properties limiting crop production in Australian sodic soils. A review. Australian Journal Soil Research, Victoria, v.32, n.1, p.13-44, 1994.

Lebron, I.; Suarez, D. L.; Alberto, F. Stability of the calcareus saline-sodic soil during reclamation. Soil Science Society American Journal, Madison, v.58, n.6, p.1753-1762, 1994.

Marano, R. Propuesta de un nuevo indicador de peligrosidad de agues bicarbonatadas-sódicas en suelos bajo riego suplementario. En: Congreso Argentino de la Ciencia del Suelo, 19, 2004, Paraná, AR. Actas ... Paraná: Asociación Argentina de la Ciencia del Suelo, 2004. CD Rom

Marano, R. P.; Pilatti, M. A. Riego suplementario con agues bicarbonatadas sódicas en Molisoles de Santa Fe. Estudio de caso: Degradación físicoquímica de un argiudol en el centro-E santafesino. En: Congreso Argentino de la Ciencia del Suelo, 18,2002, Puerto Madryn. Actas... Puerto Madryn: Asociación Argentina de la Ciencia del Suelo, 2002. CD Rom.

Mendenhall, W.; Scheaffer, R.; Wackerly, D. Estadística matemática con aplicaciones. California: Grupo Editorial Iberoamericana, 1986, 751p.

Peinemann, N.; Díaz Zorita, M.; Villamil, M. B.; Lusarreta, H.; Grunewald, D. Consecuencias del riego complementario sobre propiedades edáficas en la llanura pampeana. Ciencia del Suelo, Buenos Aires, v.16, n.1, p.39-42, 1998.

Pilatti, M.; Marano, R.; de Orellana, J. Riego suplementario con agues bicarbonatadas sódicas en Molisoles de Santa Fe. Estudio de caso: Sodificación y alcalinización. En: Congreso Argentino de la Ciencia del Suelo, 18, 2002, Puerto Madryn. Actas... Puerto Madryn: Asociación Argentina de la Ciencia del Suelo, 2002. CD Rom.

Rahman, B. A.; Malcom O. J.; Rengasamy, P. Soil estructure degradation and mellowing of compacted soils by saline-sodic solutions. Soil Science Society American Journal, Madison, v.60, n.2, p.583-588, 1996.

Raine, S. Effect of water quality on soil structure and infiltration under furrow irrigation. Plant and Soil, Netherlands, v.23, n.2, p.55-60. 2004.

Resendiz, D. Relevance of Atterberg limits in evaluating piping and breaching potential. Dispersive clay, related piping, and erosion in geotechnical projects. ASTM. Spec. Publ. 623. Filadelfia: ASTM, Ed. J.L. Sherard; R.S, Decker., 1977, p.341-353. 
Rhoades, J. D. Quality of water for irrigation. Soil Science, USA, v.113, p.277-284, 1972.

Richards, L. A. 1954. Diagnosis and improvement of saline and alcali soil. Washington DC: USDA, 1954. 160p. Agricultural Handbook, n.60.

SAGPyA. Secretaría de Agricultura, Ganadería, Pesca y Alimentación de la Nación Argentina, Subsecretaría de Agricultura, Ganadería y Forestación. Riego complementario en sistemas sustentables de la Región Pampeana. Buenos Aires: SAGPyA, 1997, 16p.

SAGPyA. Secretaría de Agricultura, Ganadería, Pesca y Alimentación de la Nación Argentina. Dirección de Producción Agrícola. Sistema de Apoyo Metodológico a los Laboratorios de Análisis de Suelos. 2.ed. Buenos Aires: SAGPyA, 2004. CD Rom
Singh, C. S.; Gupta, S. K.; Sewa, R. 1996. Assesement and management of poors quality waters for crop production: A simulation model (SWAM). Agricultural Water Management, Amsterdan, v.30, p.25-40, 1996

Soil Survey Staff. Soil taxonomy agricultural handbook, 436. Washington DC: USDA, 1999, 869p.

Topp, G. C.; Reynolds, W. D. Advances in measurement of soil physical properties: Bringing theory into practice. Madison: Soil Science Society of America, 1992, 288p. SSSA Special Publication n.30

van Olphen, H. An introduction to clay colloid chemistry. 2.ed. New York: Wiley - Interscience, 1977, 234p. 